\title{
Impact of non-random vibrations in Mössbauer rotor experiments testing time dilation
}

\author{
Y. Friedman ${ }^{1}$, I. Nowik ${ }^{2}$, I. Felner ${ }^{2}$, J. M. Steiner ${ }^{1}$, E. Yudkin ${ }^{1}$, S. Livshitz ${ }^{1}$, H.-C. Wille ${ }^{3}$, G.

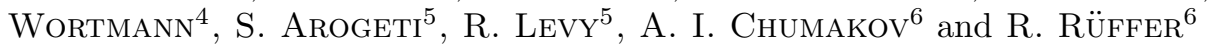 \\ 1 Jerusalem College of Technology, P.O.B. 16031 Jerusalem 91160, Israel \\ 2 Racah Institute of Physics, Hebrew University, Jerusalem 91904, Israel \\ 3 Deutsches Elektronen-Synchrotron, Notkestr. 85, D-22607 Hamburg, Germany \\ 4 Dep. Physik, Universität Paderborn, Warburger Str. 100, D-33098 Paderborn, Germany \\ 5 Ben-Gurion University of the Negev, P.O.B 653, Beer-Sheva 8410501, Israel \\ 6 European Synchrotron Radiation Facility, BP 220, F-38043 Grenoble, France
}

PACS $03.30 .+\mathrm{p}-$ Special relativity

PACS 76.80.+y - Mössbauer effect; other -ray spectroscopy

PACS 46.40.-f - Vibrations and mechanical waves

\begin{abstract}
All experiments testing time dilation by measuring the spectral shift of a rotating Mössbauer absorber assume that vibrations do not affect the spectral shift because of their purely random nature and claim that the observed shift is due to time dilation only. Our recent experiment using the Synchrotron Mössbauer Source at ESRF revealed, however, a shift due to the non-random periodic vibration patterns caused by the rotor/bearing system. These patterns fit the predictions of the Jeffcott model for such a system with non-zero eccentricity. We have calculated this shift due to the non-random vibrations and the resulting relative shift between two states when the acceleration of the absorber is anti-parallel and parallel to the source. This relative shift exhibits the same behavior as the observed relative shift. Hence, the effect of the spectral shift due to vibrations cannot be ignored in any Mössbauer rotor experiments for testing time dilation. Recommendations for improvement of future rotor experiments testing time dilation are presented.
\end{abstract}

Introduction. - Einstein's theory of Special Relativity (SR) predicts [1] a time dilation of a moving object by the known $\gamma$ factor. For regular velocities the $\gamma$ factor is very close to one, so one needs very accurate means to measure it. Since time is directly connected to frequency, the frequency absorption spectrum of a moving absorber will be shifted according to this time dilation. A natural way to detect this shift is by using a uniformly rotating absorber. This shift is equal to the Transverse Doppler (TD) shift, as predicted by SR.

Any frequency absorption spectral line for a single line absorber is approximately a Lorentzian centered at the resonant frequency. A typical absorption line is obtained by placing the source on a transducer and moving it with a small velocity in the direction of the absorber. By the Longitudinal Doppler (LD) shift due to the relative velocity of the source and the absorber, the radiation reaching the absorber has different frequencies covering the entire neighbourhood of the resonant frequency of the source. The absorption line $L\left(v_{s}\right)$ of the source velocity $v_{s}$ is: $A\left(v_{s}\right)=a \Gamma^{2} /\left(\left(v_{s}-v_{0}\right)^{2}+\Gamma^{2}\right)$, where $v_{0}$ is the velocity corresponding to this resonant frequency, called the resonant velocity. Thus, for a single line absorber, the shift between two absorption lines is the difference between their resonant velocities.

In Mössbauer absorption spectroscopy, an absorber is exposed to a beam of $\gamma$-rays near the resonant frequency, and a detector measures the intensity of the beam transmitted through the absorber. The TD shift causes a difference in resonant velocities of an absorber at rest and a rotating one. Although this difference is tiny, the extremely narrow spectral line-width of a Mössbauer absorption lines correspond to large changes in absorbance and allow to detect this tiny difference.

After the discovery of the Mössbauer effect in 1958, quantitative measurements of the relativistic time dila- 
tion, expressed by the TD shift, were carried out in the 1960s, [2] - [3]. In these experiments the Mössbauer source was fixed to the center of a fast rotating disk with an absorber placed on its rim, and the predicted TD shift was based on the absorption value at the frequency of the source. In the analysis of these experiments, it was assumed that the absorption line of the rotating absorber had a Lorentzian shape with the same width as its spectrum at rest, and that the spectral shift of this line is due to time dilation only.

Kündig, in his ingenious experiment [4], was the only one who obtained experimentally a few points of the Mössbauer absorption line of a rotating absorber by placing the source of a transducer at the center of a rotating disk. He discovered that there is a broadening of this line during the rotation and he attributed it to vibrations in the rotor. He also assumed that this broadening has no influence on the position of the resonant line. This assumption is true for random vibrations of the rotor, but he already suspected that there could be non-random vibrations caused by the bearing.

This broadening observed by Kündig shows that the shift cannot be derived from the absorption value at a single frequency, assumed by others. Based on this discovery, Kholmetskii et al [5] and [6] measured the individual absorption values of two Mössbauer absorbers each at the source frequency. The first value was used to estimate the broadening and the second to measure the TD shift. They assumed that the broadening is the same for both absorbers. The time dilation observed in these experiments differ significantly from the one predicted by SR, and they explain this deviation by the YARK theory [6]. They also assumed however that the vibrations are random and do not affect the spectral shift.

Even nowadays, absorption spectral lines are usually obtained by placing the Mössbauer source on a transducer. For technical reasons, it is very complicated to keep the balance of a fast rotating disk, if there are moving parts on it. Thus, one is forced to have the transducer outside the rotating disk. In this case it was claimed [7] and confirmed experimentally [8] that the absorption line of a rotating Mössbauer absorber gets broader during the rotation for a source outside the rotating disk. This necessitates the use of a strong Mössbauer source with a capability to be focused to the center of the disk. The recently invented Synchrotron Mössbauer Source (SMS) [9] at the Nuclear Resonance Beamline ID18 of ESRF together with the KBoptics [10] to focus this beam, was the ideal choice.

During July 2014, Friedman et al. [8] performed experiment HC-1361 at this beamline at ESRF and obtained for the first time the entire resonant line of a rotating absorber. It was shown that in this setup this line is shifted by the so called alignment shift (AS). The AS is shown to be proportional to the distance of the beam from the axis of rotation, the rotation frequency and it changes sign with the change of direction of rotation. This implies that in order to assign a true physical meaning of an observed shift one needs a way to neutralise or measure parameters which are not affected by the AS. The TD shift could be obtained from the average observed shifts of two runs in opposite directions at the same sufficiently large rotation frequency. On the other hand, if in the same run, one separates the absorption in two states (a) and (b) when the acceleration is anti-parallel and parallel to the SMS radiation (Figure 1), the absorption lines for each state are affected by the same AS. Thus, their difference, called the relative shift, is not affected by the AS.

Based on the experiences in the above experiment, a new experiment HC-1898 was performed in July 2015 at the same beamline ID18 with the stainless steel Mössbauer absorber replaced by an enriched single line absorber to increase significantly the count rate. An adjustable slit which allows a gradual better focusing of the beam was also used. Most importantly, an electronic computerized system providing on-line information on the vibrations of the disk was incorporated. This experiment confirmed that within the given setup and a fixed rotation frequency, there is indeed a statistically significant relative shift which remains stable for up to a 12 hour run.

The experiment revealed however, that one must also consider the significant effect of the periodic, non-random vibrations of the rotor/bearing system on the spectral shift. The forced steady state response of a Jeffcott rotor/bearing system with non-zero eccentricity [11] predicts periodic vibrations and radial velocities for each point of the rotating disk. Using two proximity sensors and an optical sensor we have shown that the vibrations of our rotor systems fits the Jefcott model. We have estimated this radial velocity and used it to calculate the relative spectral shift due to these vibrations. For each run, this calculated relative shift exhibits the same behaviour both in magnitude and sign as the observed relative shift.

Hence, any Mössbauer rotor experiment testing time dilation using absorption spectral shifts must consider the vibrations effect on this shift. We propose how this could be achieved.

Experimental setup. - In this experiment we obtained absorption lines of a rotating Mössbauer absorber. In order to obtain a significant count rate in such an experiment, a strong Mössbauer source was needed. The SMS at ESRF proved to be the ideal choice for such experiment as it provided a ${ }^{57} \mathrm{Fe}$ resonant radiation at $14.4 \mathrm{keV}$ within a bandwidth of $15 \mathrm{neV}$. In contrast to a common radioactive source, the beam emitted by the SMS is almost fully resonant and fully polarized, has high brilliance and can be focused by use of KB-optics to a $10 \mu \mathrm{m} \times 5 \mu \mathrm{m}$ spot size. The beam from the SMS passes the KB-optics, hits the fast rotating absorber and is finally detected by the detector $D$ diametrically opposed to the SMS (Figure 1)

We used a rotor system with a $50 \mathrm{~mm}$ radius disk, made of Titanium $6 \mathrm{Al}-4 \mathrm{v}$, designed for rotation frequencies of up to $1 \mathrm{kHz}$. A semicircular shaped single-line absorber $A$ was placed on the rim of the disk and a semicircular mark $M$ 


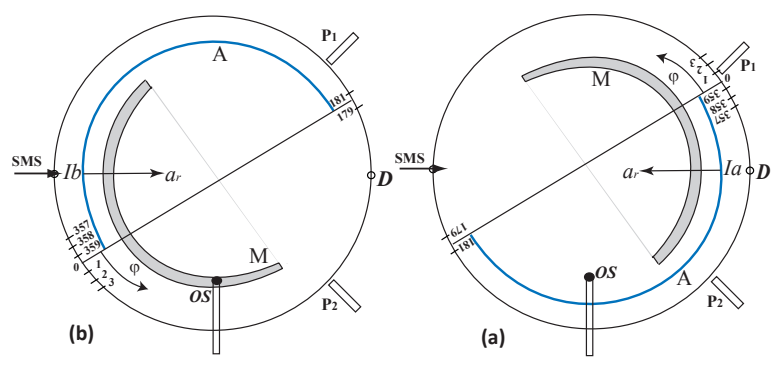

Figure 1: Two states of the disk and sensors

shifted by $90^{\circ}$ with respect to the absorber was marked on the top of the disk. The disk was lowered inside a vacuum chamber and driven by a high-speed air-bearing rotor, produced by Colibri Spindles Ltd., Israel, located outside of the chamber (Figure 2).

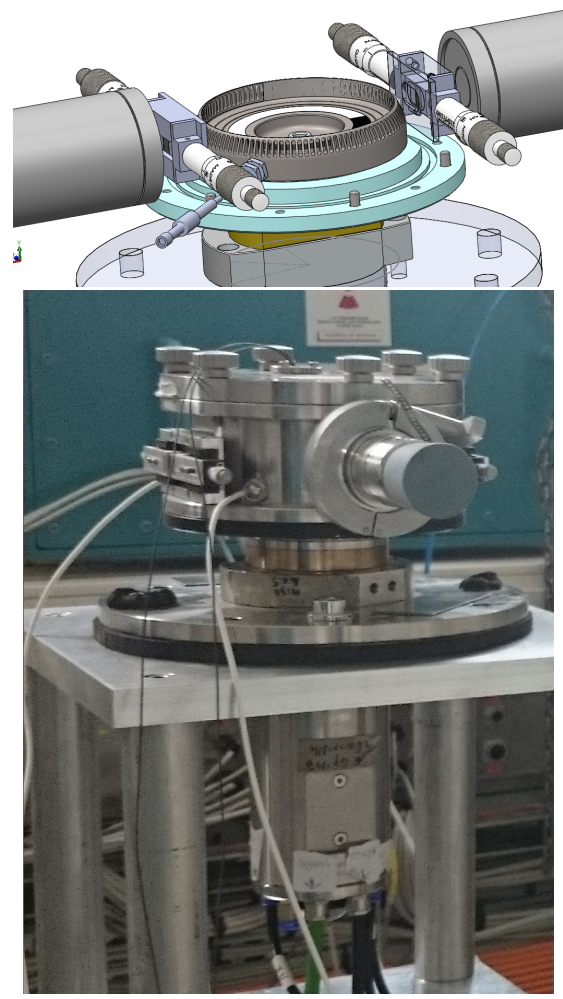

Figure 2: (top) Scetch of the disk with a semicircular absorber, placed on the shaded part of the rim of the disk. (bottom) Photo of the rotor at Beamline ID18.

Vacuum conditions are essential for high-speed rotation, low friction and acoustic noise. The chamber had two openings each of width $2 \mathrm{~mm}$ covered by transparent Mylar to allow the beam to cross the chamber. The rotor system was designed to maintain constant rotational frequencies in both directions. An enriched ${ }^{57} \mathrm{Fe}$ (95\%) $\mathrm{K}_{4} \mathrm{Fe}(\mathrm{CN})_{6} \cdot 3 \mathrm{H}_{2} \mathrm{O}$ single line material prepared by applying the procedure described in (Ganguil, 1998) was used as the Mössbauer absorber.

The beam from the SMS crosses the disk along its diameter. As the disk rotates, the radial acceleration $a_{r}$ of the absorber A is alternately directed parallel (state (b)) and antiparallel (state (a)) to the photon wavevector, yielding opposite signs for the postulated additional frequency shift due to this acceleration (Figure 1). An optical sensor $O S$ facing the disk mounted on the top of the chamber was used to identify state (a) when $O S$ does not face $M$, and (b) when $O S$ faces $M$. The SMS system was modified in order to obtain simultaneously the spectra corresponding to each state. The absorption lines were measured by recording the transmitted intensity as a function of the Doppler detuning of the SMS, as in (Potapkin et al. 2012).

Two proximity sensors $P_{1}$ and $P_{2}$ (by Micro-Epsilon Ltd.) were placed orthogonal to one another in order to measure the radial displacement of the disk (Figure 1). Collecting simultaneously the data from these sensors and the $O S$ allowed us to monitor vibration at any point of the disk.

The relative spectral shift. - The beamtime assigned to us allowed a total of 39 runs (most of them used for the initial setup, alignment and testing of the system) with a gradual increase of the rotation frequency from run to run up to $300 \mathrm{~Hz}$. During each run we obtained the resonant line for each of the above mentioned states (a) and (b) and calculated the relative shift by subtracting the shift of the spectral line at state (b) from the one at state (a). With rotation frequencies up to $100 \mathrm{~Hz}$, the two spectra were almost identical, as expected (Figure 3, top).

With a clockwise (CW) rotation of $200 \mathrm{~Hz}$, in runs assigned as 30, 31 and 32 of about 6 hours each, we observed relative shifts of $0.45 \pm 0.28 \mathrm{~mm} / \mathrm{s}, 0.42 \pm 0.27 \mathrm{~mm} / \mathrm{s}$ and $0.41 \pm 0.27 \mathrm{~mm} / \mathrm{s}$, respectively. These results show the existence of a statistically significant non-zero and constant relative shift in these runs. The corresponding average shifts in these runs were $-0.80 \mathrm{~mm} / \mathrm{s},-0.79 \mathrm{~mm} / \mathrm{s}$ and $-0.41 \mathrm{~mm} / \mathrm{s}$, respectively. Although the average shift differed from run to run probably due to the alignment, the relative shift remained almost the same for all the three runs. This indicates that the relative shift is not sensitive to the alignment of the system, as it was expected.

We then increased the frequency to $300 \mathrm{~Hz}$. Run 33 for 4 hours in $\mathrm{CW}$ direction produced even a larger relative shift $0.56 \pm 0.43 \mathrm{~mm} / \mathrm{s}$, as expected (Friedman et al. 2015). The most significant data came from run 39 for 12 hours in counter clockwise (CCW) direction at this frequency. During this time scale, the relative shift remained almost constant with value $-2.0 \pm 0.45 \mathrm{~mm} / \mathrm{s}$ (Figure 3 , bottom), however, we also observed that during this run there was a drift in the alignment of the system which caused broadening of the spectra, but did not affect the relative shift.

We did not expect such a large discrepancy in the relative shifts due to the change in direction of rotation at the same rotation frequency of $300 \mathrm{~Hz}$. Even more surprising was the change of its sign. This revealed to us the 

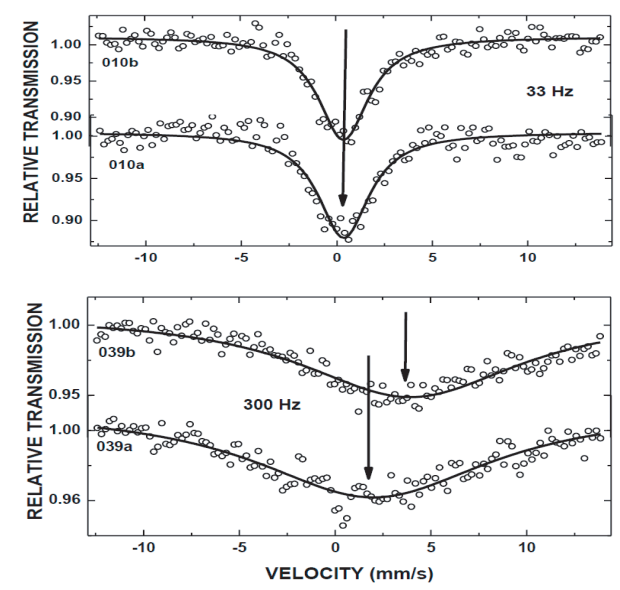

Figure 3: Spectra at $33 \mathrm{~Hz}$ and $300 \mathrm{~Hz}$, converted to average linear velocity form, for the states (a) and (b) of the absorber.

effect of some other intrinsic phenomena, not noticed previously. Since we used the same source, the same optics, the same absorber and the same rotor system, the primary reason for such discrepancies was the change in the non-random vibration patterns from $\mathrm{CW}$ to $\mathrm{CCW}$ rotations. Fortunately, the two proximity sensors $P_{1}, P_{2}$ with angle $90^{\circ}$ between them in Figure 1, and the optical sensor monitored simultaneously the radial displacement and the angular position of the disk throughout the experiment. Using the data from these sensors, we analyzed the vibration patterns and their effect on the relative shift.

Vibration analysis of a rotating disk. - At any time $t$, the two proximity sensors measured their respective distances $p_{1}(t), p_{2}(t)$ to the disk rim. Assuming that the disk is rigid, these distances define the position of the geometric center of the disk at any time. The spectral analysis of $p_{1}(t), p_{2}(t)$ are shown in Figure 4.

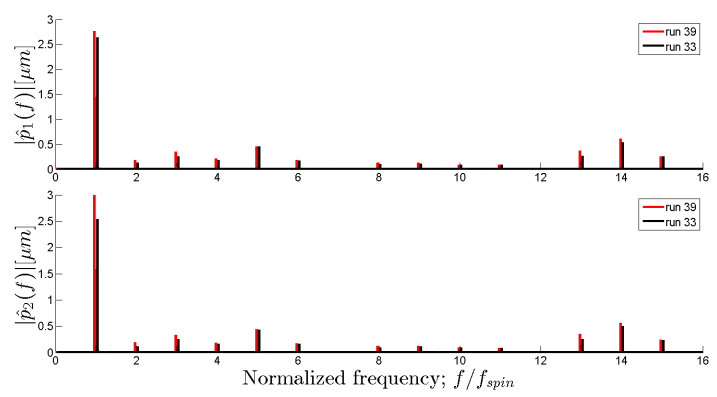

Figure 4: Amplitude spectra of $p_{1}(t)$ and $p_{2}(t)$ for CW (run 33 , black) and CCW (run 39, gray) rotation of $300 \mathrm{~Hz}$

These graphs show the amplitude spectra of the proximity sensor data $p_{1}(t)$ and $p_{2}(t)$, respectively. The frequency axis is normalized with respect to the rotation speed. The two different colors correspond to runs 33 and 39 with a small shift intentionally added to allow distinction be- tween the lines. For both sensors, the highest amplitude matches exactly the rotor rotational speed $300 \mathrm{~Hz}$ (or 1 on the normalized axis), proving that the vibrations are nonrandom with period matching the rotational frequency. The frequency of the higher harmonics with much smaller amplitudes are also multiples of the rotor rotational speed, probably resulting from the complex dynamics of the airbearing rotor system.

The non-randomness and the periodicity of the vibration patterns could be explained by the use of the Jeffcott rotor/bearing model for synchronous vibration of a rotor system with eccentricity of the rotating disk. The Jeffcott rotor model (Yoon et al, 2013) describes the motion of an unbalanced rotating disk affected by the bearing system by the following equations (resulting from the Newton's second law)

$$
\begin{aligned}
m \ddot{x}_{C}+c \dot{x}_{C}+k x_{C} & =m e \omega^{2} \cos (\omega t) \\
m \ddot{y}_{C}+c \dot{y}_{C}+k y_{C} & =m e \omega^{2} \sin (\omega t)
\end{aligned}
$$

were $\left(x_{C}, y_{C}\right)$ represents the coordinates of the position of the disk geometric center, $e$ is the eccentricity (i.e., the shift of the center of mass from the disk geometric center), $\omega$ is the constant rotational frequency and other parameters $m, k$ and $c$ represent the disk mass (the shaft is assumed massless), the air bearing stiffness and the air bearing dumping, respectively.

The steady state solutions of this model for the $x$ direction, which is relevant to us, is

$$
x_{C}(t)=P \cos (\omega t+\beta),
$$

where the amplitude $P$ and phase angle $\beta$ are given by

$$
P=\frac{e \omega^{2}}{\sqrt{\left(k / m-\omega^{2}\right)^{2}+(c \omega / m)^{2}}}
$$

and

$$
\tan \beta=\frac{-c \omega / m}{k / m-\omega^{2}} .
$$

According to the Jeffcott rotor model, formula (1) predicts that the dominant frequency of $x_{C}(t)$ matches exactly the rotational frequency $\omega$ of the disk, as stated above. Furthermore, formula (3) predicts that the phase shift $\beta$ in the vibration patterns changes sign with the change in the direction of rotation. This explains the possibility of the observed sign change of the relative shift due to the change in the direction of rotation.

In order to calculate the effect of vibrations on the relative shift one needs to associate the vibrations to states (a) and (b) of the system. Since these states are defined by the angular position of the disk, the radial distances $p_{1}(t), p_{2}(t)$ at time $t$ must be converted to the corresponding radial distances $p_{1}(\varphi), p_{2}(\varphi)$ at the position $\varphi$ on the disk. We chose to mark the position of any point on the disk by its angle $\varphi$ measured CCW from the end of the semicircular absorber $A$ (Figure 1). 
We collected data with a constant sampling rate simultaneously from the optical sensor $O S$, used now for identifying the position on the disk, and the two proximity sensors $P_{1}, P_{2}$. We wrote a computer program to associate the radial displacements $p_{1}(\varphi), p_{2}(\varphi)$, measured by $P_{1}$ and $P_{2}$ to the position $\varphi$ at the time of measurement, taking into account the direction of rotation. To achieve this, we identify for each revolution, the exit time $t_{0}$ of $M$ from the sight of $O S$ and use the formula $\varphi(t)=\omega\left(t-t_{0}\right)+\varphi_{0}$ where $\varphi_{0}$ is the angular position of $P_{1}$ or $P_{2}$ at time $t_{0}$. Using the data from a large number of revolutions, we obtained the average radial displacement for each point $\varphi$ on the disk. Such averaging eliminates the contribution of the random vibrations.

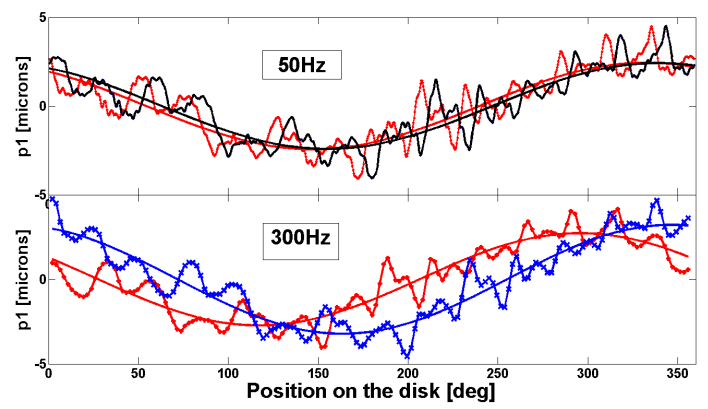

Figure 5: Comparison of $\mathrm{CW}$ and $\mathrm{CCW}$ average radial displacements at $50 \mathrm{~Hz}$ (top) and $300 \mathrm{~Hz}$ (bottom) and their fit by Jeffcott rotor model

Figure 5 reveals that average radial displacements fit approximately the solution expressed by (1). Moreover, according to this model, changing rotation from $\mathrm{CW}$ to $\mathrm{CCW}$ at the same rotational frequency, the amplitude $P$ given by (2) is not affected while the phase $\beta$ given by (3) changes its sign, resulting in a phase shift $2 \beta$ between the lines in the two directions. These predictions are confirmed in Figure 5 for both $50 \mathrm{~Hz}$ and $300 \mathrm{~Hz}$ rotations. Moreover, formula (3) predicts that the phase shift $2 \beta$ between the two directions increases non-linearly with $\omega$. As we see from the figure, the observed phase shift corresponding to the $2 \beta$ shift, predicted by the model, at $300 \mathrm{~Hz}$ is approximately $48^{\circ}$.

Effect of rotor vibrations. - The crucial information that is of relevance to time dilation experiments is whether the rotor vibration has a significant effect on the spectral shift and ultimately on the observed relative spectral shift. Define the points of incidence - $I_{a}, I_{b}$ as the points where the beam strikes the absorber in states (a), (b) respectively (see Figure 1). The main contribution to such a shift comes from the LD shift defined by the component of the velocity of the absorber at the point of incidence. Since we focused the beam to the axis of rotation, the direction of the beam is along the line joining the axis of rotation and the points of incidence $I_{a}$ or $I_{b}$, the radial direction.

The spectral shift can be calculated by averaging the radial velocity of the absorber at the point of incidence over the time of the data collection (the run). This radial velocity of a given point on the disk $v_{r}(t)$ at a particular time $t$ can be estimated by measuring the radial distances of this point $r(t \pm \Delta t)$ for a short time interval $\Delta t$ and is given by

$$
v_{r}(t)=\frac{r(t+\Delta t)-r(t-\Delta t)}{2 \Delta t} .
$$

Thus, in order to be able to calculate the shift caused by vibrations we need to measure the radial distance of any point of the absorber at two positions near the point of incidence corresponding to times $t \pm \Delta t$.

Denote by $t$ the time at which a point on the disk $\varphi$ is at the incident point $I_{a}$. Then, for a $\mathrm{CW}$ rotation of period $T$, the radial displacements $p_{1}(\varphi), p_{2}(\varphi)$ are $r(t-$ $\Delta t), r(t+\Delta t)$ respectively, with $\Delta t=T / 8$. Hence from (4),

$$
v_{r}(\varphi)=\frac{p_{2}(\varphi)-p_{1}(\varphi)}{2 \Delta t} .
$$

For a CCW rotation, the order is reversed and

$$
v_{r}(\varphi)=\frac{p_{1}(\varphi)-p_{2}(\varphi)}{2 \Delta t} .
$$

Thus, we have the information for calculating the radial velocity $v_{r}(\varphi)$ for the time when the point of incidence is $I_{a}$. Assuming perfect rigidity of the disk, if the point of incidence is $I_{b}$ the radial velocity of the absorber is $v_{r}(\varphi+\pi)$.

The spectral shift caused by the vibrations can be calculated as follows: Monitor the vibrations for several thousand periods. Since this shift is defined by the mean radial velocity at each point $\varphi$ on the disk over the whole run, it can be estimated by the average of $v_{r}(\varphi)$, calculated by the above formulae over this sample. Figure 6 presents this mean radial velocity due to vibrations at $300 \mathrm{~Hz} \mathrm{CW}$ and $\mathrm{CCW}$ rotations.

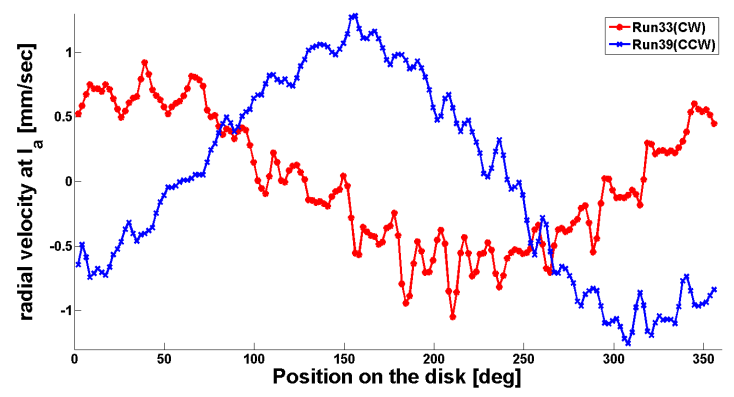

Figure 6: Mean radial velocity $v_{r}(\varphi)$ in $\mathrm{mm} / \mathrm{s}$ at the point of incidence $I_{a}$ due to $300 \mathrm{~Hz} \mathrm{CW}$ (gray) and CCW (black) vibrations

From Figure 6 we see that $v_{r}(\varphi)$ also follows a sinusoidal pattern, predicted by differentiation of formula (1). Changing the direction of rotation causes a phase shift of $180^{\circ}$ which combined with displacement $2 \beta$ shift predicts a $180^{\circ}-2 \beta$ shift between the two $v_{r}(\varphi)$ curves. In our 
case this shift was approximately $127^{\circ}$. The random deviations from this pattern average out to zero and do not contribute to the shift.

We estimated $v_{r}(\varphi)$ at $I_{a}$. Thus, for $\varphi \in\left[0,180^{\circ}\right]$ this velocity corresponds to state (b), while for $\varphi \in\left[180^{\circ}, 360^{\circ}\right]$ to state (a), see Figure 1. So the calculated shift due to vibrations in states (b), (a) is the average of $v_{r}(\varphi)$ in the intervals $\left[0,180^{\circ}\right],\left[180^{\circ}, 360^{\circ}\right]$, respectively.

We calculated the relative shift due to the rotor vibrations by subtracting the shift in state (b) from that in state (a). The calculated relative shift for run 33 (CW) was $0.42 \mathrm{~mm} / \mathrm{s}$ and for run $39(\mathrm{CCW})$ was $-0.66 \mathrm{~mm} / \mathrm{s}$. In both cases the observed relative shifts are consistent with these calculated shifts. For run 33 the observed relative spectral shift is close to the calculated relative shift due to vibrations. For run 39, both the change in sign as well as the increase in magnitude of the observed relative shift as compared to run 33 are consistent with that which is due to the rotor vibrations. However, for run 39 the observed relative shift is significantly larger than the calculated vibrational one.

Conclusions. - Our experiment HC-1898 using the SMS at ID18 of ESRF, as our previous experiment HC1361 at the same facility, obtained the entire resonant absorption line of a single line Mössbauer absorber on the rim of a rotating disk. The experiment revealed, as expected, that within the given setup and a fixed rotation frequency, there is a stable statistically significant relative shift, not affected by the alignment, between the absorption lines in the two states when the acceleration of the absorber is anti-parallel and parallel to the SMS radiation.

We discovered, however that in addition to the purely random vibrations, the rotor/bearing system generates also non-random periodic patterns which affect the relative spectral shift. These patterns were shown to fit the predictions of the Jeffcott model of a rotor/bearing with non-zero eccentricity. This model predicts a non-random periodic radial velocity with the same frequency as the rotation frequency for each point of the rotating disk, which in turn generates a spectral shift. Hence, the assumption of the purely random nature of vibrations in Mössbauer rotor experiments is not valid.

Unlike in the experiments performed by others, in our experiment the source was outside the disk. This allowed us to obtain the full absorption spectrum. Having the source on the disk itself indeed minimizes the effect of the vibrations; however, it yields only a single point and not the full absorption line that we obtained. This necessitates assumptions on the behavior of the full spectrum which may not be valid. For example, the assumption of [6] that the distance between the source and the absorber remains constant during the rotation contradicts the broadening of the absorption line already observed by Kündig.

Using two proximity sensors and an optical sensor we monitored the vibrations, eliminated the purely random contribution, estimated the radial velocity due to their non-random contribution and calculated its effect on the observed relative shift. For our rotor system, this calculated relative shift exhibits the same behavior both in magnitude and sign, as the observed relative shift.

A Mössbauer rotor experiment with a chance to prove the validity of Einstein's (or alternative) time dilation formulae, must have a method to measure the vibrations of the rotor system and quantify its effect on the spectral shift. In this paper we proposed a way how this could be achieved.

Finally, we propose a few recommendations desirable for future experiments:

1. Use of a "vibrationless" rotor system which ensures that for some frequencies the spectral shift due to the rotor vibrations is much smaller than the one caused by time dilation (the object one is looking for).

2 . Use of a system to estimate or measure directly the radial velocity of the absorber at the point(s) of incidence.

3. Use of computer simulators for rotating disks to estimate the relative velocity between the source and absorber on (or outside) a non-perfectly rigid disk and its influence on the spectral shift.

4. Perform the experiment for each rotational frequency in both directions, in order to detect and eliminate some side-effects.

5. For experiments with the source at the center of the disk, place several Mössbauer detectors along the circumference of the disk, to enable the partial detection of the effect of vibrations at different angles of the bearing system.

We thank the referee and the support of the Jerusalem College of Technology and ESRF at Grenoble, France.

References

[1] A. Einstein, Annalen der Physik, 17 (1905) 891

[2] H. J. HAY et al, Phys. Rev. Lett., 4 (1960) 165

[3] T. E. Cranshaw, J. P. Schiffer and P. A. Egelstaff, Phys. Rev. Lett., 4 (1960) 163

[4] W. KüNDig, Phys. Rev., 129 (1963) 2371

[5] A. L. Kholmetskil et al, Phys. Scr., 79 (2009) 065007

[6] T. Yarman, A. L. Kholmetski, and M. Arik, Eur. Jour. Plus, 130 (2015) 191

[7] Y. Friedman and I. Nowik, Phys. Scr., 85 (2012) 065702

[8] Y. Friedman et al, J. Synch. Rad., 22 (2015) 723

[9] V. Potapkin et al, J. Synch. Rad., 19 (2012) 559

[10] P. Kirkpatrick and A. V. Baez, J. Opt. Soc. Am., 38 (1948) 766

[11] S.Y. Yoon ET AL., Control of Surge in Centrifugal Compressors by Active Magnetic Bearings, Advances in Industrial Control (Springer-Verlag London) 2013

[12] S.Ganguil, S. DAS And M. Bhattacharya, J. Radioanal. Nuclear Chem., 232 (1998) 229 